\title{
Raman scattering efficiency of graphene
}

\author{
P. Klar, ${ }^{1}$ E. Lidorikis, ${ }^{2}$ A. Eckmann, ${ }^{3}$ I. A. Verzhbitskiy, ${ }^{1}$ A. C. Ferrari, ${ }^{4}$ and C. Casiraghi ${ }^{1,3}$ \\ ${ }^{1}$ Physics Department, Free University Berlin, Berlin, Germany \\ ${ }^{2}$ Materials Science and Engineering Department, University of Ioannina, Ioannina, Greece \\ ${ }^{3}$ School of Chemistry, University of Manchester, Manchester, United Kingdom \\ ${ }^{4}$ Cambridge Graphene Centre, Cambridge University, 9 JJ Thomson Avenue, Cambridge, United Kingdom
}

(Received 14 December 2012; revised manuscript received 5 April 2013; published 24 May 2013)

\begin{abstract}
We determine the Raman scattering efficiency of the $G$ and $2 D$ peaks in graphene. Three substrates are used: silicon covered with 300 or $90 \mathrm{~nm}$ oxide, and calcium fluoride $\left(\mathrm{CaF}_{2}\right) . \mathrm{On} \mathrm{Si} / \mathrm{SiO}_{x}$, the areas of the $G$ and $2 D$ peak show a strong dependence on the substrate due to interference effects, while on $\mathrm{CaF}_{2}$ no significant dependence is detected. Unintentional doping is reduced by placing graphene on $\mathrm{CaF}_{2}$. We determine the Raman scattering efficiency by comparison with the $322 \mathrm{~cm}^{-1}$ peak area of $\mathrm{CaF}_{2}$. At $2.41 \mathrm{eV}$, the Raman efficiency of the $G$ peak is $\sim 200 \times 10^{-5} \mathrm{~m}^{-1} \mathrm{Sr}^{-1}$, and changes with the excitation energy to the power of 4 . The $2 D$ Raman efficiency is at least one order of magnitude higher than that of the $G$ peak, with a different excitation energy dependence.
\end{abstract}

DOI: 10.1103/PhysRevB.87.205435

PACS number(s): 78.30.-j, 78.67.Wj, 81.05.ue

\section{INTRODUCTION}

Graphene attracts enormous interest because of its unique properties. ${ }^{1-7}$ Near-ballistic transport at room temperature and high mobility ${ }^{5,6,8-11}$ make it a potential material for nanoelectronics, ${ }^{12-16}$ especially for high frequency applications. Furthermore, its optical and mechanical properties are ideal for micro- and nanomechanical systems, thin-film transistors, transparent and conductive composites and electrodes, and photonics. ${ }^{17-24}$

Elastic and inelastic light scattering are powerful tools for investigating graphene. ${ }^{25-27}$ Raman spectroscopy allows monitoring of doping, defects, strain, disorder, chemical modifications, and edges. ${ }^{28-42}$

Besides these practical applications, Raman spectroscopy in graphitic systems is interesting per se because it involves resonant conditions, defect-induced processes and strong electron-phonon coupling. ${ }^{42-47}$

The determination of the Raman scattering efficiency in carbon-based materials has not been thus far the subject of many investigations. The dependence of the Raman efficiency on the excitation energy was studied in diamond, ${ }^{48,49}$ graphite, ${ }^{50,51}$ nanographites,${ }^{52}$ fullerene solutions, ${ }^{53}$ hydrogenated amorphous carbon, ${ }^{54-56}$ nanodiamond films, ${ }^{57}$ and carbon nanotubes. ${ }^{58,59}$ The absolute value of the Raman scattering efficiency of highly oriented pyrolytic graphite (HOPG) was reported in two seminal works: the one of Wada et al., ${ }^{51}$ who used diamond as a reference scatterer, and that of Sinha et al., ${ }^{50}$ who used silicon as a reference scatterer. They found the $G$ peak Raman scattering efficiency measured at $2.41 \mathrm{eV}$ to be $\sim 300-400 \times 10^{-5} \mathrm{~m}^{-1} \mathrm{Sr}^{-1}$ and $\sim 800 \times 10^{-5} \mathrm{~m}^{-1} \mathrm{Sr}^{-1}$, respectively. ${ }^{50,51}$

Knowledge of the Raman scattering efficiency is of great importance because it can provide detailed information on the electron-phonon interaction, such as the deformation potentials, ${ }^{60}$ the two phonon absorption cross section, ${ }^{61}$ electro-optic coefficients, ${ }^{62}$ electron-phonon interaction under hydrostatic pressure, ${ }^{63,64}$ to cite a few. Furthermore, the Raman efficiency dependence on excitation energy can probe singularities in the dielectric constant, ${ }^{60}$ since an enhancement in the Raman efficiency is expected whenever the incident or scattered energy approaches these resonances. ${ }^{65-71}$ In the case of carbon-based materials, a quantitative knowledge of Raman efficiency would allow one to evaluate the relative amount of graphitelike to diamondlike bonds in amorphous and disordered carbons. ${ }^{54,57,72}$ In the case of carbon nanotubes, this would allow one to use Raman spectroscopy as a fully quantitative tool to evaluate the abundance of each chirality. ${ }^{58}$ However, an accurate determination of the Raman efficiency faces various hurdles, such as corrections for sample absorption, collection angle, spectral sensitivity, and surface conditions. ${ }^{60}$ These have not been explicitly discussed in most previous works on graphite and amorphous carbons. ${ }^{50,54,57}$ In particular, the spectrometer sensitivity can strongly affect the apparent Raman efficiency dependence on excitation energy.

All $s p^{2}$ bonded carbons show common features in their Raman spectra, the so-called $G$ and $D$ peaks, around 1580 and $1360 \mathrm{~cm}^{-1}$. $^{73}$ The $G$ peak corresponds to the $E_{2 g}$ phonon at the Brillouin zone center ( $\Gamma$ point). The $D$ peak is due to the breathing modes of six-atom rings and requires a defect for its activation. ${ }^{45,74,75}$ It comes from TO phonons around the $\mathbf{K}$ point, ${ }^{74,75}$ is active by double resonance (DR), ${ }^{45}$ and is strongly dispersive with excitation energy due to a Kohn anomaly at K. ${ }^{43}$ The activation process for the $D$ peak is intervalley: (i) a laser induced excitation of an electron/hole pair; (ii) electronphonon scattering with an exchanged momentum $\mathbf{q} \sim \mathbf{K}$; (iii) defect scattering; (iv) electron-hole recombination. DR can also happen as an intravalley process, i.e., connecting two points belonging to the same cone around $\mathbf{K}$ ( or $\mathbf{K}^{\prime}$ ). This gives the so-called $D^{\prime}$ peak, which is at $\sim 1620 \mathrm{~cm}^{-1}$ in defected graphite measured at $514 \mathrm{~nm}$. The $2 D$ peak is the second order of the $D$ peak. This is a single peak in single layer graphene (SLG), whereas it splits in four in bilayer graphene (BLG), reflecting the evolution of the band structure. ${ }^{25}$ The $2 D^{\prime}$ peak is the second order of the $D^{\prime}$ peak. Since both $2 D$ and $2 D^{\prime}$ originate from a process where momentum conservation is satisfied by two phonons with opposite wave vectors ( $\mathbf{q}$ and $-\mathbf{q}$ ), they do not require defects for their activation, and are thus always present. Indeed, high quality graphene shows the $G, 2 D$, and $2 D^{\prime}$, but not $D$ and $\mathrm{D}^{\prime} .25$ The $2 D$ and $2 D^{\prime}$ peaks are triple resonant. ${ }^{42,46,47}$ This means that all intermediate electronic states are real. As a consequence, 
two-phonon Raman spectroscopy is sensitive to the dynamics of the photoexcited electron-hole pair, in particular, to the scattering processes it can undergo. ${ }^{44,47}$

Note that one-phonon modes in defect-free samples can be Raman active only if their symmetry is correct and their wave vector is zero (i.e., obey the fundamental Raman selection rule). In SLG only the $G$ peak satisfies these requirements. ${ }^{42}$ The energies of the intermediate states are given by the difference in energies of electrons in the empty $\pi^{*}$ and filled $\pi$ bands, $\epsilon_{\mathbf{k}}^{\pi^{*}}-\epsilon_{\mathbf{k}}^{\pi}$ (with $\mathbf{k}$ the electronic wave vector), with or without the phonon energy, $\hbar \Omega_{\mathbf{q}=\mathbf{0}} .{ }^{42}$ The decay rate of the intermediate states is given by the sum of the scattering rates of the electron in the $\pi^{*}$ band, $2 \gamma_{\mathbf{k}}^{\pi^{*}} / \hbar$, and of the hole in the $\pi$ band, $2 \gamma_{k}^{\pi} / \hbar$. The contribution from the phonon decay is typically smaller. ${ }^{42}$ Counterintuitively, the electronic wave vectors k mostly contributing to the matrix element for the $G$ peak are not just such that the excitation energies $\epsilon_{\mathbf{k}}^{\pi^{*}}-\epsilon_{\mathbf{k}}^{\pi}$ lie within an interval $\sim \gamma$ from $\hbar \omega_{L}$ or $\hbar \omega_{L}-\hbar \Omega_{\mathbf{q}=0}$, with $\hbar \omega_{L}$ being the incident laser photon energy. Instead, they are such that $\left|\epsilon_{\mathbf{k}}^{\pi^{*}}-\epsilon_{\mathbf{k}}^{\pi}-\hbar \omega_{L}\right|$ can be of the order of $\hbar \omega_{L}$ itself, and there are strong cancellations in the sum over k. ${ }^{76}$ These cancellations correspond to destructive quantum interference. In fact, this interference can be controlled externally. Indeed, occupations of electronic states can be changed by doping and, since transitions from an empty state or to a filled state are impossible due to Pauli blocking, doping can effectively exclude some regions of $\mathbf{k}$ from contributing to the matrix element. ${ }^{42}$ Due to suppression of destructive interference, this leads to an increase of the $G$ peak intensity at high doping levels, as was predicted in Ref. 76 and observed in Refs. 77 and 78. Thus, unlike the $2 D$ peak, in undoped or low-doped samples, the $G$ peak arises from nonresonant processes, and only at extremely high doping, not dealt with in this paper, and anyway difficult to achieve in any standard experiment, do resonant contributions have a role.

In graphitic materials the intensity of the Raman features strongly depends on the amount of disorder. ${ }^{52,72,74,75,79-84}$ Single-crystal graphite and exfoliated graphene can have negligible $D$ peak intensities, and thus be structurally nearly perfect. Therefore, these are ideal for an accurate investigation of their Raman scattering efficiency. Here we determine the Raman scattering efficiency of single-crystal graphite (SCG) and SLG. We find that the Raman intensity of SLG on $\mathrm{Si} / \mathrm{SiO}_{x}$ is modulated by the substrate, reaching a maximum at $\sim 530 \mathrm{~nm}$ excitation under our experimental conditions, when graphene is placed on $300 \mathrm{~nm}$ silicon oxide. This is due to interference enhanced Raman effects. This is confirmed by measuring SLG on a transparent substrate $\left(\mathrm{CaF}_{2}\right)$, where no intensity modulation with the excitation energy is found. Therefore, we use the Raman intensities of graphene measured on $\mathrm{CaF}_{2}$ to determine the Raman efficiency by the sample substitution method (Sec. II). We find that the Raman scattering efficiency of the $G$ peak $[d S / d \Omega(G)]$ is $\sim 200 \times 10^{-5}$ $\mathrm{m}^{-1} \mathrm{Sr}^{-1}$ at $2.41 \mathrm{eV}$. In our samples, the $2 D$ peak Raman scattering efficiency $[d S / d \Omega(2 D)]$ is much higher than the Raman scattering efficiency of the first order line of silicon and gallium arsenide, measured at $2.41 \mathrm{eV}$. This is quite unusual, since normally the overtones intensities are much smaller than the first order peaks. ${ }^{67}$ This large value for the Raman scattering efficiency of the $2 D$ peak, compared with other overtones efficiencies, clearly shows that the area of the 2D peak is given by resonant contributions. ${ }^{44,46,85,86}$

This paper is organized as follows. Section II describes the experiential setup. Section III presents background concepts on the Raman scattering efficiency and the sample substitution method. Section IV discusses the Raman scattering efficiency dependence on substrate and excitation energy and gives the absolute Raman efficiencies of $G$ and $2 D$ peaks, measured at $2.41 \mathrm{eV}$.

\section{EXPERIMENT}

Graphene flakes are produced by micromechanical cleavage of SCG (Nacional de Grafite LTDA) using Nitto tape. ${ }^{87}$ Three substrates are used: silicon covered with $300 \mathrm{~nm}$ and $90 \mathrm{~nm}$ silicon oxide (IDB Technologies LTD) and $\mathrm{CaF}_{2}[111]$ (crystal $\mathrm{GmbH}$ ). The number of layers are estimated by a combination of Rayleigh and Raman scattering. ${ }^{25,26}$ The oxide thickness of the silicon substrates used here allows graphene to be optically visible, ${ }^{26}$ while the optical contrast of graphene on $\mathrm{CaF}_{2}$ is very weak, but enough to identify single graphene layers by optical microscopy and Raman spectroscopy. ${ }^{26,88}$ Fresh and clean SCG surfaces are prepared by cleaving SCG flakes by Nitto tape before measuring the spectra.

Raman spectroscopy is performed with various spectrometers: a Renishaw single monochromator, equipped with 488 , 514, and $633 \mathrm{~nm}$ excitation energies, a Dilor triple monochromator (Horiba-Jobin Yvon), equipped with excitation lines from $647 \mathrm{~nm}$ to $457 \mathrm{~nm}$, and a HORIBA XploRA Confocal Raman Spectrometer, equipped with $785 \mathrm{~nm}$ laser wavelength. In all cases the microscope objective has a numerical aperture (NA) of 0.9. Raman maps are taken with a Witec single monochromator, equipped with $633 \mathrm{~nm}$ excitation. This spectrometer features also an atomic force microscope (AFM), used here to study the surface properties of graphene deposited on $\mathrm{CaF}_{2}$. All Raman spectra are recorded in backscattering, with incident light normal to the sample surface.

The Raman spectrum of graphene consists of a set of distinct peaks. Each characterized by its position width, height, and area. We use the following notation: ${ }^{42} I$ for peak height, $A$ for peak area, Pos for peak position, FWHM for the full width at half maximum. So, e.g., $I(G)$ is the height of the $G$ peak, $A(G)$ its area, $\operatorname{FWHM}(G)$ the full width at half maximum and $\operatorname{Pos}(G)$ its position. The frequency-integrated area under each peak represents the probability of the whole process. It is more robust with respect to various perturbations of the phonon states than width and height. ${ }^{44}$ The measured peaks are fitted with Lorentzians. For BLG, four Lorentzians are used to fit the $2 D$ peak, while in thicker layers the $2 D$ peak is fitted with two Lorentzians, with the sum of the integrated areas of the subpeaks taken to represent the overall $2 D$ strength. Several measurements are performed both on the same spot and different spots on the same sample. We only consider flakes much larger than the laser spot size $\left(\sim 1 \mu \mathrm{m}^{2}\right)$, to avoid edge effects. ${ }^{33}$ All measurements are performed with power on the sample below $0.6 \mathrm{~mW}$.

Sometimes peaks at $\sim 1350,1450$, and $1530 \mathrm{~cm}^{-1}$ are seen; see Fig. 1. We attribute them to glue and tape residuals, as confirmed by performing Raman spectroscopy directly on the tape used to exfoliate SCG. In this case, the peak at 


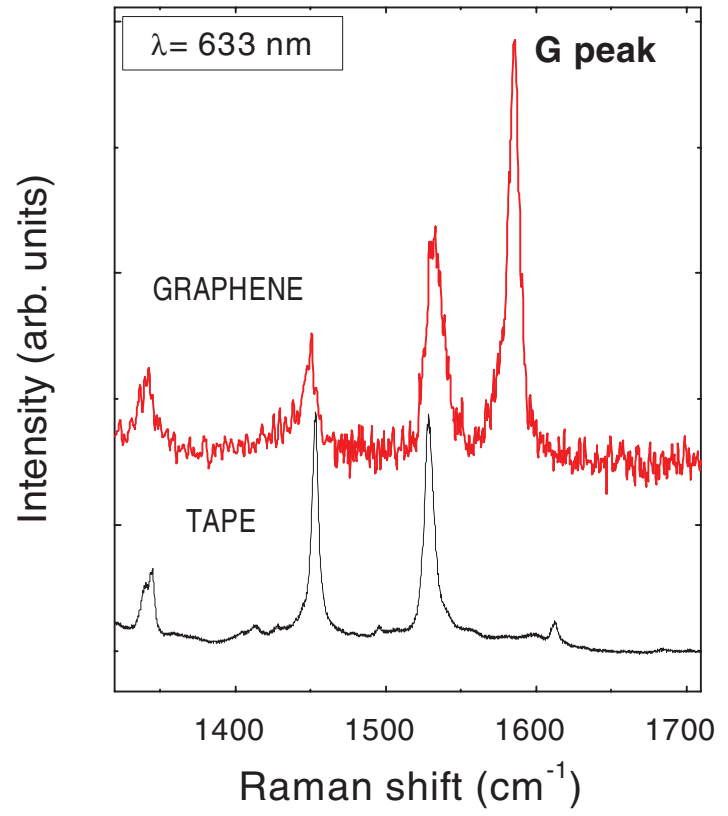

FIG. 1. (Color online) Raman spectra of SLG with Nitto tape residuals and Nitto tape, measured at $633 \mathrm{~nm}$.

$\sim 1350 \mathrm{~cm}^{-1}$ cannot be attributed to the $D$ peak. Note that the $D$ peak changes position with excitation energy, moving to $\sim 1320 \mathrm{~cm}^{-1}$, when measured at $633 \mathrm{~nm},{ }^{39}$ unlike the tape peaks.

The typical approach to measure the Raman efficiency is to compare the Raman intensity of the sample under study with that of another sample used as a reference (substitutional method). ${ }^{60} \mathrm{~A}$ commonly used standard scatterer is liquid benzene, since its Raman efficiency and its dependence on excitation energy are well known. ${ }^{89}$ However, several corrections are needed, such as those to account for the solid angle and reflection losses, and spectrometer sensitivity. An alternative method compares the Raman and Brillouin spectra from the same sample, measured at the same time in the same setup, and uses the Brillouin data as reference. ${ }^{90-93}$ This approach relies on the fact that Brillouin scattering cross sections can be expressed in terms of elasto-optic coefficients and other macroscopic quantities, which in turn can be determined using independent techniques. ${ }^{91}$ This was used to measure the Raman efficiency of the first order silicon peak ${ }^{91}$ and the second order of strontium titanate $\left(\mathrm{SrTiO}_{3}\right),{ }^{93}$ potassium iodide $(\mathrm{KI}),{ }^{93}$ potassium bromide $(\mathrm{KBr}),{ }^{93}$ and potassium chloride $(\mathrm{KCl}) .{ }^{92}$ Here, we employ the substitutional method, using $\mathrm{CaF}_{2}$ as a reference scatterer (Sec. III).

\section{BACKGROUND}

\section{A. Raman scattering efficiency}

The Raman scattering efficiency $d S / d \Omega$ has dimensions of an inverse length, and is defined as the ratio between scattered and incident power, for a unit solid angle and optical probe depth $(L):^{60}$

$$
\frac{d S}{d \Omega}=\frac{I_{\text {ins }}}{\Delta \Omega_{\text {ins }}} \frac{\hbar \omega}{P_{\text {ins }} L},
$$

where $I_{\text {ins }}, \Delta \Omega_{\text {ins }}$, and $P_{\text {ins }}$ are the number of photons, solid angle collection, and laser power inside the crystal. $L$ is (i) for a transparent sample, either the focal length or the thickness of the sample, whichever the smaller ${ }^{60}$ and (ii) $L=1 /\left(\alpha_{i}+\alpha_{s}\right)$, if the sample is opaque, where $\alpha_{i}$ and $\alpha_{s}$ are the absorption coefficients measured at incident and scattered energies. ${ }^{60,71,94-96}$ However, the signal measured in a Raman experiment is the number of scattered photons outside the crystal, within a solid collection angle $\Delta \Omega$. Thus, to get the Raman scattering efficiency from the peak's area, Eq. (1) needs to be corrected for light refraction, and reflection losses incurred when light enters and leaves the crystal: ${ }^{97}$

$$
\begin{aligned}
& A=I_{\text {ins }}\left(1-R_{s}\right), \\
& P_{\text {ins }}=P_{0}\left(1-R_{i}\right), \\
& \Delta \Omega_{\text {ins }} \approx \Delta \Omega / n_{s}^{2},
\end{aligned}
$$

where $R_{i}$ and $R_{s}$ are the reflectance measured at the incident and scattered frequency, $n_{s}$ the refractive index at the scattered frequency, and $P_{0}$ is the laser power. Inserting Eqs. (2)-(4) into Eq. (1), we get:

$$
A=\frac{\left(1-R_{i}\right)\left(1-R_{s}\right)}{\hbar \omega} \frac{P_{0} \Delta \Omega L}{n_{s}^{2}} \frac{d S}{d \Omega} .
$$

This is the relation between Raman peak area and scattering efficiency. Within a microscopic description, the Raman scattering efficiency is given by ${ }^{98}$

$$
\frac{d S}{d \Omega}=\omega^{4} \frac{2 \hbar N^{2}}{\rho c^{4} \omega_{\mathrm{ph}}}\left(n_{\mathrm{ph}}+1\right) \sum_{j}\left|\mathbf{e}_{\mathbf{i}} R_{j} \mathbf{e}_{\mathbf{s}}\right|^{2},
$$

where $c$ is the light speed, $\rho$ is the density, $N$ is the number of primitive cells per unit volume, $\omega_{\mathrm{ph}}$ is the phonon frequency, and $n_{\mathrm{ph}}$ is the phonon occupation number. We approximated $\left(\omega-\omega_{\mathrm{ph}}\right) \sim \omega$. $\mathbf{e}_{\mathbf{i}}$ and $\mathbf{e}_{\mathbf{s}}$ are the unit vector representing the polarization of the incident and scattered light. $R_{j}$ is the Raman tensor of the phonon $j$, containing the tensor element $a$ (the Raman polarizability), with: ${ }^{60}$

$$
a^{2}=V\left|\frac{d \chi\left(\omega_{i}\right)}{d \xi}\right|^{2},
$$

where $V$ is the volume, $\chi$ is the susceptibility, and $\xi$ is the normal mode coordinate.

For a second order overtone process, Eq. (6) has the following form:

$$
\frac{d S}{d \Omega} \sim \omega^{4} \sum_{i, \mathbf{q}}\left|\mathbf{e}_{\mathbf{s}} \frac{\partial^{2} \chi}{\partial \xi_{i}(\mathbf{q}) \partial \xi_{i}(-\mathbf{q})} \mathbf{e}_{\mathbf{i}}\right|^{2} .
$$

By putting Eqs. (6) and (8) into Eq. (5), the first order and overtone Raman peak area can be described directly as a function of the excitation energy and the Raman tensor, respectively. Note the dependence of the Raman scattering efficiency on the excitation frequency (e.g., energy) to the power of 4 in Eqs. (6) and (8). Since all the other parameters in Eqs. (6) and (8) are not expected to vary with the excitation energy, then the Raman efficiency should increase with $\omega^{4}$. However, this is not always true: the dependence of the Raman efficiency on excitation frequency over and above $\omega^{4}$ is expected to be small or null only if the excitation energy is far from any singularity of the dielectric function, i.e., only when the process is nonresonant. Under resonance conditions, the 
Raman tensor element strongly changes with the excitation frequency, so the Raman efficiency does not follow the $\omega^{4}$ law. ${ }^{60}$ In semiconductors this is typically observed when the excitation energy is close to the optical gap of the material: under this condition a strong enhancement of the Raman peaks areas is observed. ${ }^{60}$ The resonance intensity profile was indeed used in the past to study the electronic structure of several semiconductors. ${ }^{60}$

\section{B. Substitutional method}

The absolute value of the Raman scattering efficiency can be measured from the Raman peaks areas by using Eq. (5). However, a Raman peak area strongly depends on the experimental setup, such as type and numerical aperture of the objective, power, integration time, and on surface conditions. E.g., Ref. 51 reported that the silicon intensities increased a factor of 2 after polishing the surface. Thus, the direct use of Eq. (5) does not provide accurate values for the Raman efficiency.

In order to measure the absolute value of the Raman scattering efficiency, we use the substitutional method. ${ }^{60}$ This consists in normalizing the Raman peak area of the sample to that of the substitutional scatterer (indicated by $*$ in the following equations) and correcting this ratio by the optical constants of the two materials. From Eq. (5), we have

$$
\frac{A}{A^{*}}=\frac{\left(1-R_{i}\right)\left(1-R_{s}\right)}{\left(1-R_{i}^{*}\right)\left(1-R_{s}^{*}\right)} \frac{n_{s}^{* 2}}{n_{s}^{2}} \frac{L}{L^{*}} \frac{d S / d \Omega}{d S^{*} / d \Omega} .
$$

Thus we can get the Raman scattering efficiency simply measuring the Raman peaks areas ratio between the two materials.

The advantage of this method is that $A / A^{*}$ is automatically corrected for the $\omega^{4}$ dependence and for the spectrometer and detector sensitivity, which strongly varies with the excitation energy. ${ }^{48}$ Every Raman spectrometer has its own sensitivity curve, depending on the type of detector, gratings, and optics used. This can be measured with a calibration lamp. In our case, we used a quartz tungsten halogen (QTH) lamp (Oriel, Newport Corp.) with a calibrated spectral irradiance between 250 and $2400 \mathrm{~nm}$. The sensitivity curve for our triple monochromator spectrometer is shown in Fig. 2. Note that the spectrometer response varies by almost one order of magnitude between 2.2 and $2.7 \mathrm{eV}$.

In order to use the substitutional method, we need to choose as a reference scatterer a material whose optical properties and Raman efficiency are very well known in the visible range. One could then use the first order of the silicon peak. However, silicon is resonant in the visible, so the silicon peak area needs to be corrected for the dispersion of its dielectric function. Thus the simplest way to get the absolute value of the Raman scattering efficiency and its energy dependence is to use a material which is not resonant in the energy range investigated. In this way any residual energy dependence of $A / A^{*}$ can be ascribed totally to the Raman tensor, since spectrometer sensitivity and $\omega^{4}$ dependence are automatically corrected and the Raman tensor of the reference material is constant, i.e.,

$$
\frac{A}{A^{*}} \sim \frac{d S / d \Omega}{d S^{*} / d \Omega} \sim \frac{\sum_{j}\left|\mathbf{e}_{\mathbf{i}} R_{j} \mathbf{e}_{\mathbf{s}}\right|}{\sum_{j}\left|\mathbf{e}_{\mathbf{i}} R_{j}^{*} \mathbf{e}_{\mathbf{s}}\right|} \sim \sum_{j}\left|\mathbf{e}_{\mathbf{i}} R_{j} \mathbf{e}_{\mathbf{s}}\right| .
$$

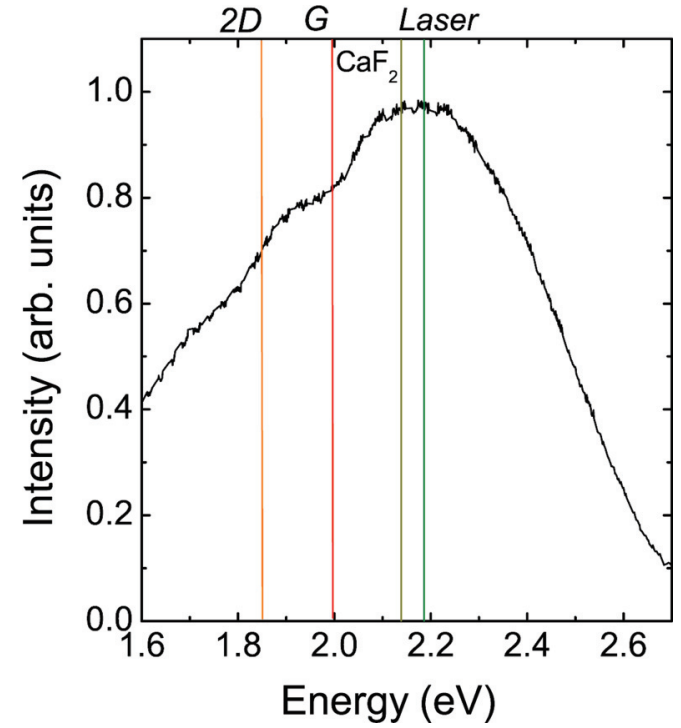

FIG. 2. (Color online) Sensitivity curve of our Dilor Raman spectrometer setup (detector, grating, and optics).

By choosing a reference material which is not resonant under the energy range investigated, the energy dependence of $A / A^{*}$ directly describes that of the Raman tensor of the material under investigation. This is a different approach with respect to the experiments based on cyclo-hexane, where the measured Raman peaks areas include also the $\omega^{4}$ dependence. $^{52}$

Here we use as reference material $\mathrm{CaF}_{2}$ : this crystal shows only one first order line at $\sim 320 \mathrm{~cm}^{-1}$, and its gap is $\sim 11 \mathrm{eV}$, well above any of our excitation energies. ${ }^{98}$ This allows one to consider the $\mathrm{CaF}_{2}$ Raman tensor constant in the range 1.8-3.8 eV, within a $16 \%$ error bar. ${ }^{98}$ The substitutional method with $\mathrm{CaF}_{2}$ was previously used to measure the Raman efficiency of other materials, such as diamond, silicon, CdS, and $\mathrm{ZnO}{ }^{67,70,98}$

Using $\mathrm{CaF}_{2}$ as a reference scatterer, we have $1-R_{i}^{*}=$ $1-R_{s}^{*}=0.97$ and $n_{s}^{*}=1.437$ in the visible range. ${ }^{88}$ The Raman scattering efficiency $d S / d \Omega\left(\mathrm{CaF}_{2}\right)$ is $\sim 4.935 \times$ $10^{-7} \mathrm{~m}^{-1} \mathrm{Sr}^{-1}$ at $2.41 \mathrm{eV} .^{98}$ Being a transparent material, we assume that the $\mathrm{CaF}_{2}$ probe length corresponds to our focus length $(\sim 1000 \mu \mathrm{m})$. Thus Eq. (10) gives

$$
\frac{A}{A^{*}}=4479 L \frac{\left(1-R_{i}\right)\left(1-R_{s}\right)}{n_{s}^{2}} \frac{d S}{d \Omega},
$$

where $L$ is in $\mathrm{nm}$ and $d S / d \Omega$ in $\mathrm{m}^{-1} \mathrm{Sr}^{-1}$.

To be effective, the substitutional method requires the measurements to be performed on the sample and on $\mathrm{CaF}_{2}$ under exactly the same conditions. For this reason we placed SLG directly on $\mathrm{CaF}_{2}$ by micromechanical exfoliation. ${ }^{87}$

Note that the position of the $\mathrm{CaF}_{2}, G$, and $2 D$ peaks span over a $\sim 0.4 \mathrm{eV}$ range, so the ratio between $G, 2 D$, and $\mathrm{CaF}_{2}$ areas needs to be corrected for the spectrometer sensitivity. As an example, we consider in detail the correction performed for an excitation energy of $2.18 \mathrm{eV}(568 \mathrm{~nm})$. The $2 D$ peak lies at $\sim 2650 \mathrm{~cm}^{-1}$. This corresponds to an energy shift of $0.33 \mathrm{eV}$. Since we measure the Stokes line, the $2 D$ peak position corresponds to an absolute energy of $2.18-0.33=1.85 \mathrm{eV}$. The $G$ peak absolute energy is then $2.18-0.2=1.99 \mathrm{eV}$. 
The $\mathrm{CaF}_{2}$ peak, used as a reference, lies at $\sim 325 \mathrm{~cm}^{-1}$, i.e., at an energy of $\sim 2.14 \mathrm{eV}$. Thus Fig. 2 shows that the measured $A(2 D)$ is underestimated, when compared to $A(G)$. Figure 2 gives a correction factor of $\sim 0.81 / 0.7=1.14$.

We now apply the substitutional method to SLG on $\mathrm{CaF}_{2}$. Equation (11) requires transmittance, penetration length, and the refractive index of SLG. Transmission measurements on suspended SLG have shown that reflectance is negligible, while absorbance and transmittance in the visible range are constant and equal to 0.023 and 0.977 , respectively. ${ }^{99}$ Since the light penetration length cannot be smaller than the SLG effective thickness $(0.33 \mathrm{~nm})$, for SLG we use $L=0.33 \mathrm{~nm}$. The refractive index of SLG was measured by ellipsometry. ${ }^{100}$ This showed that one can use for graphene the refractive index in the basal plane of graphite, within $15 \%$ error. ${ }^{100}$ The refractive index in the basal plane of graphite can be taken as almost constant in the visible range and it is $n \simeq$ $2.69+i 1.45 .^{101}$ We then get

$$
\frac{A_{\mathrm{SLG}}}{A^{*}}=204 \frac{d S}{d \Omega_{\mathrm{SLG}}} .
$$

We now consider SCG. From the refractive index, the reflectance of graphite is $\sim 0.3$ at normal incidence, ${ }^{101}$ and its absorption coefficient $\alpha \sim 0.0341 \mathrm{~nm}^{-1}$, almost constant in the visible range. ${ }^{51,101}$ For SCG, we also need to consider the strong absorbance, so $L \simeq 1 / 2 \alpha \simeq 15$ nm. ${ }^{97}$ Thus Eq. (9) gives

$$
\frac{A_{\mathrm{SCG}}}{A^{*}}=4549 \frac{d S}{d \Omega_{\mathrm{SCG}}} .
$$

Note that $A_{\mathrm{SLG}} / A_{\mathrm{SCG}}$ does not correspond to $d S / d \Omega_{\mathrm{SLG}} /$ $d S / d \Omega_{\mathrm{SCG}}$, because of the different reflectance and penetration length in graphene and graphite.

As we will show in the next section, $A_{\mathrm{SCG}} / A_{\mathrm{SLG}}$ can be strongly modulated by the excitation energy because of the interference effects produced by the $\mathrm{Si} / \mathrm{SiO}_{x}$ substrate.

\section{RESULTS AND DISCUSSION}

\section{A. $\mathrm{Si} / \mathrm{SiO}{ }_{x}$ substrate}

Figure 3 plots $A(G)$ for graphene deposited on $\mathrm{Si} / 300 \mathrm{SiO}_{x}$ as a function of the number of layers $(N)$ of a flake, measured at (a) $633 \mathrm{~nm}$, (b) $514.5 \mathrm{~nm}$, and (c) $488 \mathrm{~nm}$, showing that $A(G)$ has a different dependence on $N$ for different excitation energies. In particular, at 488 and $514 \mathrm{~nm}, A(G)_{\mathrm{SLG}}$ is similar to $A(G)_{\mathrm{SCG}}$, while at $633 \mathrm{~nm}, A(G)_{\mathrm{SLG}}$ is $\sim 10$ times smaller than $A(G)_{\text {SCG }}$.

Figure 4 plots $A(2 D)$ as a function of $N$ deposited on $\mathrm{Si} / 300 \mathrm{SiO}_{x}$, measured at (a) $633 \mathrm{~nm}$ and (b) $488 \mathrm{~nm}$. Note the effect of doping on $A(2 D)$. Indeed, one needs to be very careful when evaluating $d S / d \Omega(2 D)$. Pristine graphene samples on $\mathrm{Si} / 300 \mathrm{SiO}_{x}$ are usually doped by charged impurities and $A(2 D)$ strongly changes with doping. ${ }^{30-32,44,102,103}$ Also, Fig. 4 implies that the $A(2 D)$ dependence on $N$ changes with the excitation energy. In particular, $A(2 D)_{\mathrm{SLG}} / A(2 D)_{\mathrm{SCG}}=0.37$ and 3.3 , when measured at 633 and $488 \mathrm{~nm}$, respectively.

We attribute this different behavior with excitation energy to interference enhanced Raman scattering. We use the transfer matrix method (TMM) ${ }^{97}$ to evaluate the effect of substrate interference effects and sample absorption on the overall

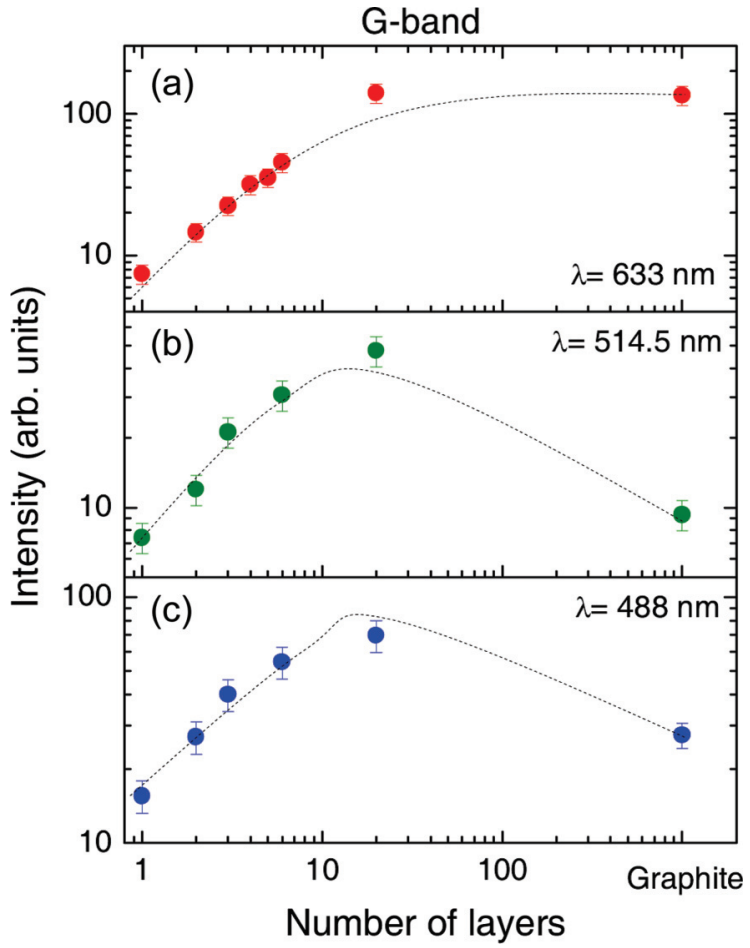

FIG. 3. (Color online) $G$ peak area, measured at (a) $633 \mathrm{~nm}$, (b) $514.5 \mathrm{~nm}$, and (c) $488 \mathrm{~nm}$ for flakes with increasing $N$, from SLG to $\mathrm{SCG}$, deposited on $\mathrm{Si} / 300 \mathrm{SiO}_{x}$. The dotted line is a guide to the eye.

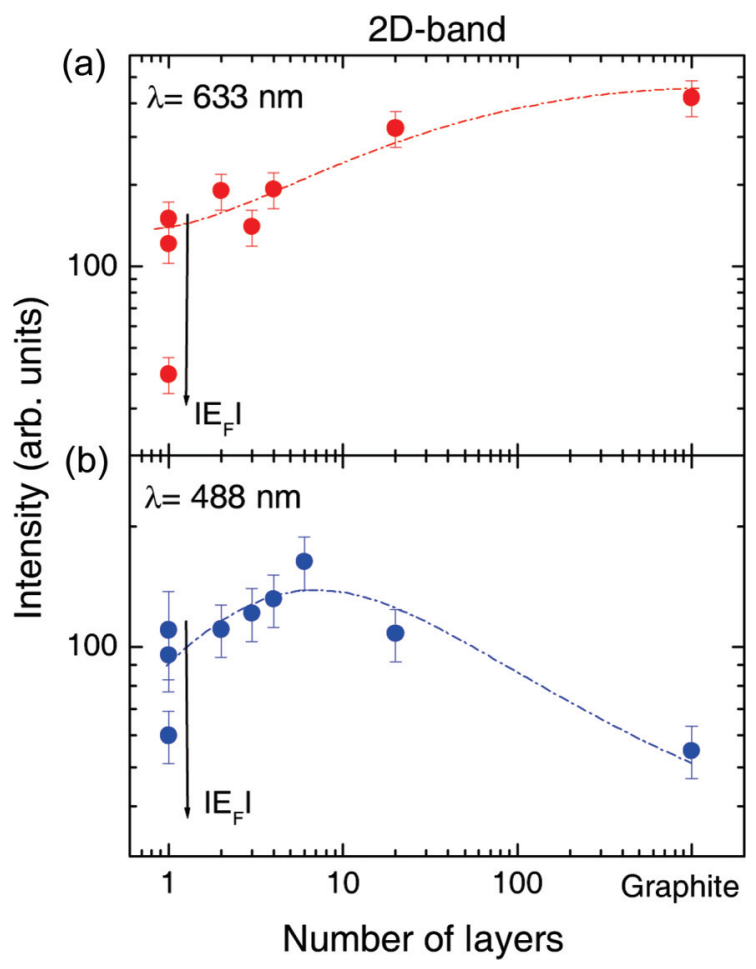

FIG. 4. (Color online) $2 D$ peak area measured at (a) $633 \mathrm{~nm}$ and (b) $488 \mathrm{~nm}$ for flakes with increasing $N$, from SLG to SCG, deposited on $\mathrm{Si} / 300 \mathrm{SiO}_{x}$. Note that $A(2 D)$ strongly depends on doping, ${ }^{44,102}$ so different SLG samples can have a large variation of $A(2 D)$ depending on the Fermi energy, $E_{F}$. The dotted line is a guide to the eye. 
Raman signal. This proceeds in two steps. First, we calculate the incident amplitude $E(x)$ in the sample as a function of depth $x$. The Raman absorption at depth $x$ is proportional to $|E(x)|^{2}$. Next we calculate the emissivity $\gamma_{R}(x)$ from depth $x$ at the corresponding Stokes-shifted frequency. The Raman intensity is then proportional to

$$
I \propto \int_{x=0}^{d}|E(x)|^{2}\left|\gamma_{R}(x)\right|^{2} d x .
$$

In the case of a nonzero NA, we perform the TMM calculation for every angle of incidence and both polarizations, so that

$$
I \propto \int_{x=0}^{d} \overline{\mathrm{E}}(x) \bar{\Gamma}_{R}(x) d x,
$$

with:

$$
\begin{gathered}
\overline{\mathrm{E}}(x)=\sum_{s, p} \int_{\theta=0}^{\pi / 2}\left|E_{\theta_{g}}(x)\right|^{2} S_{p} f(\theta) \sin \theta d \theta, \\
\bar{\Gamma}_{R}(x)=\int_{\theta=0}^{\pi / 2}\left|\gamma_{R, \theta_{g}}(x)\right|^{2} f(\theta) \cos \theta \sin \theta d \theta,
\end{gathered}
$$

where the angle $\theta_{g}$ inside the sample is related to the incident angle $\theta$ through Snell's law $\sin \theta_{g}=\sin \theta / n_{g}$, with $n_{g}$ the graphene's index of refraction and $S_{p}$ is 1 or $\left|\cos \theta_{g}\right|^{2}$ for $s$ - or $p$-polarized incidence, respectively. In Eq. (16), we assumed for simplicity that the emitting dipole is parallel to the surface and that the emission is $s$-polarized. The extra cosine in the emission integral is to enforce a Lambertian $\propto \cos \theta$ far field distribution, and the angular weight $f(\theta)$ is determined by the NA: $f(\theta)=e^{-2 \sin ^{2} \theta / \mathrm{NA}^{2}}$. Our measurements are done with $\mathrm{NA}=0.9$. For comparison, we also plot results for $\mathrm{NA}=0$ and $\mathrm{NA}=0.6$. The indexes of refraction of $\mathrm{SiO}_{2}$ and $\mathrm{Si}$ are those used in Ref. 26.

We then calculate $A(2 D)$ and $A(G)$ as a function of $N$, for three different excitation wavelengths, each for three different NA. $A(G)$, normalized to the corresponding graphite signal, is plotted in Fig. 5. We note that for $N A=0.6$ the calculations reproduce well our experimental results. This could mean that the laser does not completely fill our objective, giving an effective $\mathrm{NA} \sim 0.6$. Under our experimental conditions, at 488 and $514 \mathrm{~nm} A(G)_{\mathrm{SLG}}$ is slightly less than $A(G)_{\mathrm{SCG}}$, with a maximum enhancement (compared to graphite) of 2.5 and 4 for $N \sim 15$ layers. In contrast, at $633 \mathrm{~nm} A(G)_{\mathrm{SLG}}$ is smaller than $A(G)_{\mathrm{SCG}}$, with at least eight layers required for the signal to reach it, and a maximum enhancement of 2.5 for $N \sim 35$ (not shown in the experimental data).

We get a similar result for $A(2 D)$, as indicated in Fig. 6. We find that at $633 \mathrm{~nm}$ and NA $=0.6$ we need $N \sim 10$ to reach $A(2 D)_{\mathrm{SCG}}$, with a maximum enhancement of a factor 1.5 for $N \sim 38$ layers. However, our calculation cannot reproduce the fine features observed for SLG and FLG, as for Fig. 6. It does, however, reproduce well the results for large $N$.

One striking effect is the different trend as a function of NA that we observe at different excitation wavelengths: at $488 \mathrm{~nm}$ there is a large increase of $A_{\mathrm{SLG}} / A_{\mathrm{SCG}}$ with increasing NA, while the opposite is found for $633 \mathrm{~nm}$. At $532 \mathrm{~nm}$, on the other hand, there is very little variation with NA. This is seen for both $G$ and $2 D$ peaks. In order to understand this behavior, we plot in Fig. 7 the normalized $A(G)$ and $A(2 D)$ as a function of excitation wavelength and NA. Note

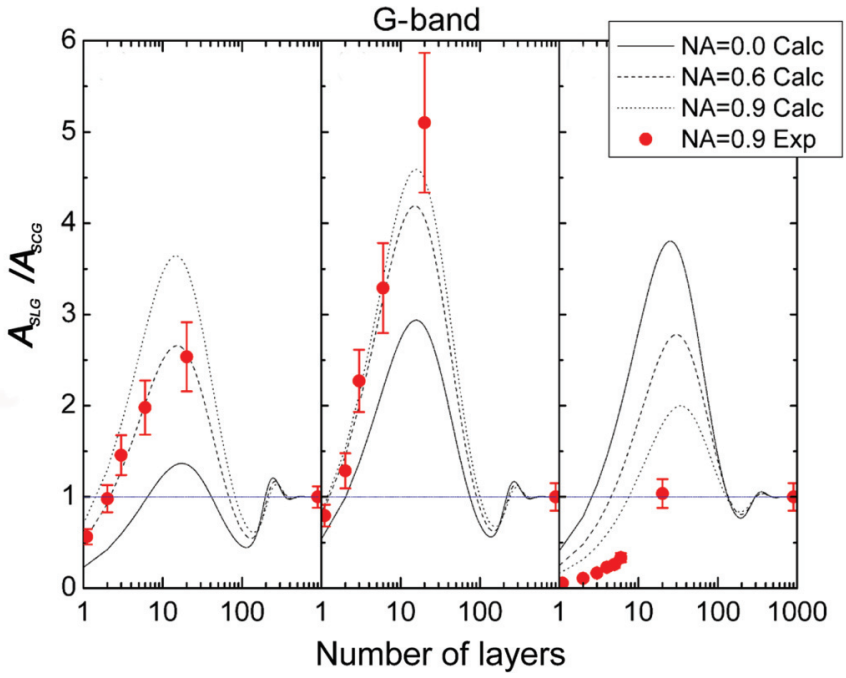

FIG. 5. (Color online) $G$-band Raman signal enhancement for graphene layers on $\mathrm{Si} / 300 \mathrm{SiO}_{x}$, compared to graphite, for three excitation wavelengths: $488 \mathrm{~nm}$ (left panel), $514 \mathrm{~nm}$ (middle panel), and $633 \mathrm{~nm}$ (right panel). Note that the trend changes as we scan different NA values.

that for the $2 D$ peak in Fig. 7(b) we scaled the graphene results by $A_{2 D}^{\text {expt }}(1) / A_{2 D}^{\text {calc }}(1) \cong 4.18$ so that a direct comparison with experiments can be made. At NA $=0$ the enhancement reaches 0.88 at $560 \mathrm{~nm}$ for $G$ and 2.9 at $545 \mathrm{~nm}$ for $2 D$. At $\mathrm{NA}=0.9$, however, the maxima shift to $515 \mathrm{~nm}$ and $500 \mathrm{~nm}$, respectively, with peak values 0.82 and 2.8. Thus, for excitation close to the maximum (where there is little slope

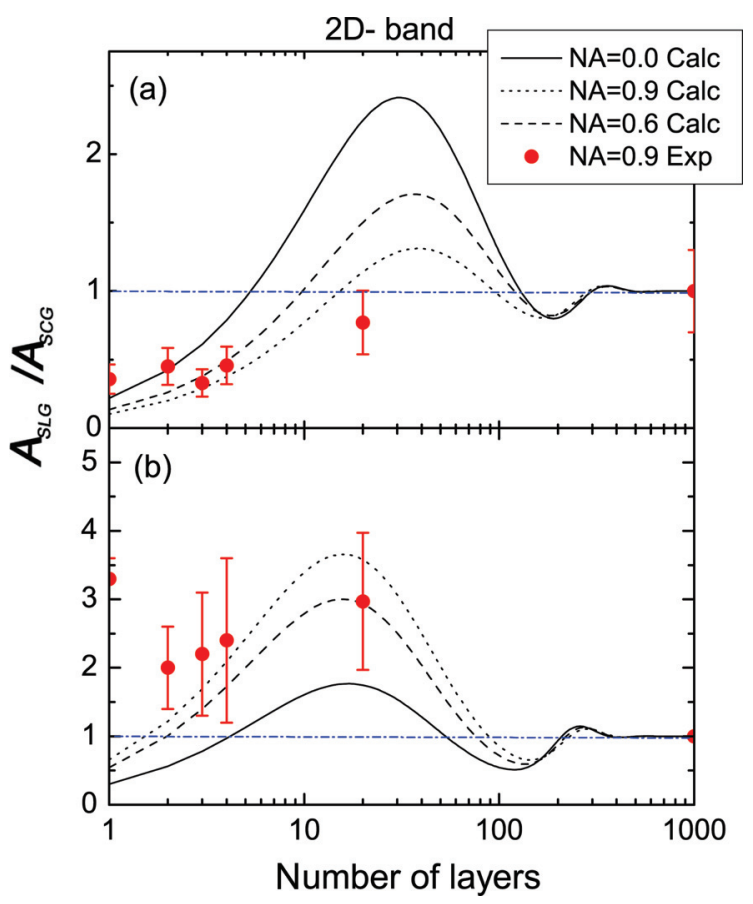

FIG. 6. (Color online) 2D-band Raman signal enhancement of graphene layers deposited on $\mathrm{Si} / 300 \mathrm{SiO}_{x}$, compared to graphite at (a) $633 \mathrm{~nm}$ and (b) $488 \mathrm{~nm}$. In each case we plot the enhancement for three different NAs. Note that the measured $A(2 D)$ do not follow the expected variation in the Raman area with sample thickness. 


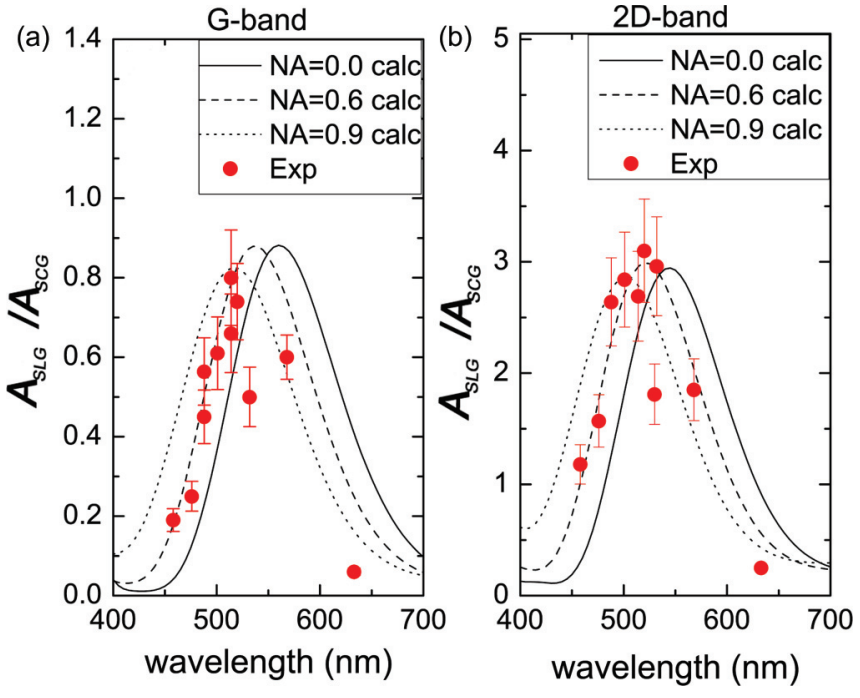

FIG. 7. (Color online) Areas of (a) $G$-band and (b) $2 D$-band for graphene on $\mathrm{Si} / 300 \mathrm{SiO}_{x}$ for three NAs. Note the effect of NA on the maximum enhancement wavelength.

with wavelength), no significant change is observed as NA increases (as is the case for the $514 \mathrm{~nm}$ excitation in Fig. 7). On the other hand, below the peak (large positive slope with wavelength) we observe significant increase in enhancement (e.g., at $488 \mathrm{~nm}$ ), while above the peak (large negative slope with wavelength) significant decrease (e.g., at $633 \mathrm{~nm}$ ). This is expected, since in all interference phenomena, as the angle increases, the resonant wavelength decreases. This underlines the crucial role of NA to fully understand the measurements.

Figure 8 plots $A(G)$ and $A(2 D)$ of $\mathrm{SLG}$ on $\mathrm{Si} / 90 \mathrm{SiO}_{x}$ measured at different excitation wavelengths. The intensities are higher than on $\mathrm{Si} / 300 \mathrm{SiO}_{x}$ and they do not show a strong dispersion with excitation wavelength and NA. The different behavior of the intensity is expected since interference enhancement strongly depends on the oxide thickness. ${ }^{26}$ Figure 8 implies that $\mathrm{Si} / 90 \mathrm{SiO}_{x}$ is indeed a better substrate in order to quickly measure a Raman spectrum, since it gives up to eight times higher intensity, depending on the excitation wavelength, compared with $\mathrm{Si} / 300 \mathrm{SiO}_{x}$.

\section{B. $\mathrm{CaF}_{2}$ substrate}

Figure 9 shows (a) the AFM picture and (b) the Raman map of graphene on $\mathrm{CaF}_{2}$ : here, the green color corresponds to regions of the sample without $D$ peak, the blue area shows regions with a $D$ peak, and the red area corresponds to the Raman spectrum of graphite. This figure implies that there are only small regions with $D$ peak, and no contamination is detected by AFM.

Figure 10 plots the Raman spectra of SLG on $\mathrm{CaF}_{2}$, measured at different excitation energies. The Raman fit parameters do not show strong variations from sample to sample, or within the same sample, and are comparable with those measured on suspended SLG. ${ }^{103}$ Thus $\mathrm{CaF}_{2}$ can be used as a substrate in order to measure the Raman efficiency of SLG, since doping, defects, and interference effects do not affect the measured Raman peaks areas, in contrast to when $\mathrm{Si} / \mathrm{SiO}_{x}$ is used as substrate.

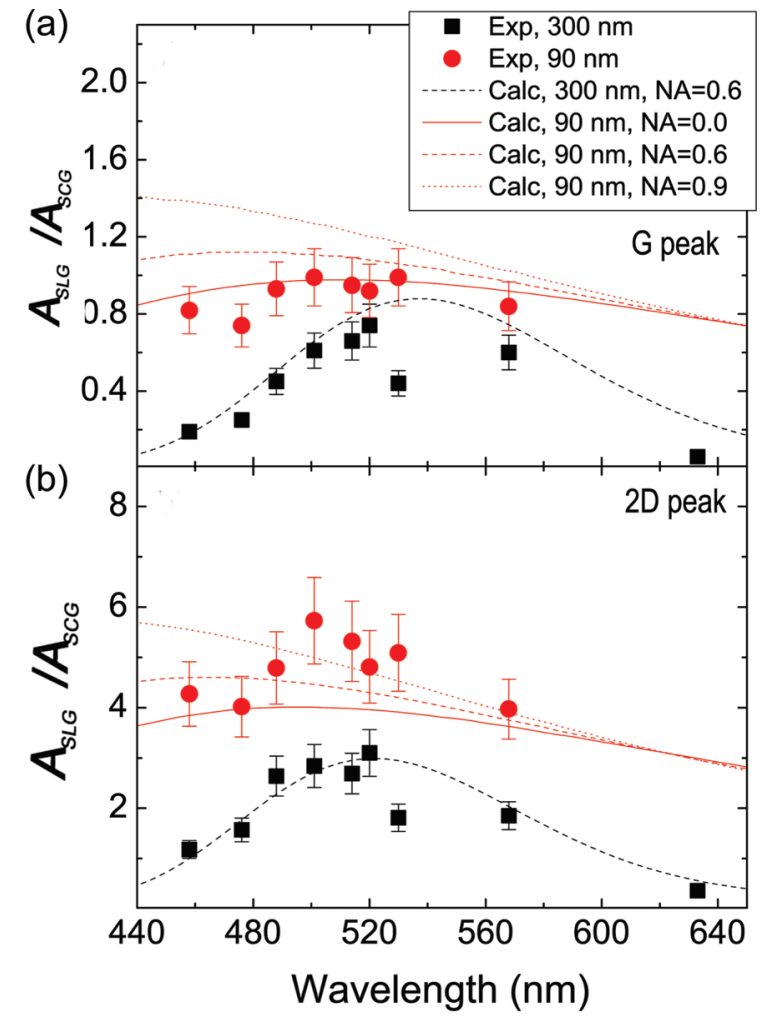

FIG. 8. (Color online) Areas of (a) $G$-band and (b) $2 D$-band for graphene on Si covered with 300 and $90 \mathrm{~nm}$ oxide.

Figure 11 plots the Raman peaks areas of SLG, compared to $\mathrm{CaF}_{2}$, as a function of excitation energy. This figure shows that $A(G)_{\mathrm{SLG}} / A\left(\mathrm{CaF}_{2}\right)$ and $A(2 D)_{\mathrm{SLG}} / A\left(\mathrm{CaF}_{2}\right)$ have a very different energy dependence. This is ascribed to the Raman tensor (Sec. III): that of the $G$ peak is constant, so the Raman scattering efficiency follows the $\omega^{4}$ law. This is an indication that the $2 D$ peak area is ruled by resonant contributions. ${ }^{42}$ In contrast, the Raman tensor of the $2 D$ peak is not constant in the energy range investigated here, so the corresponding Raman scattering does not follow the $\omega^{4}$ law. This agrees with previous results obtained for nanocrystalline graphite. ${ }^{52}$
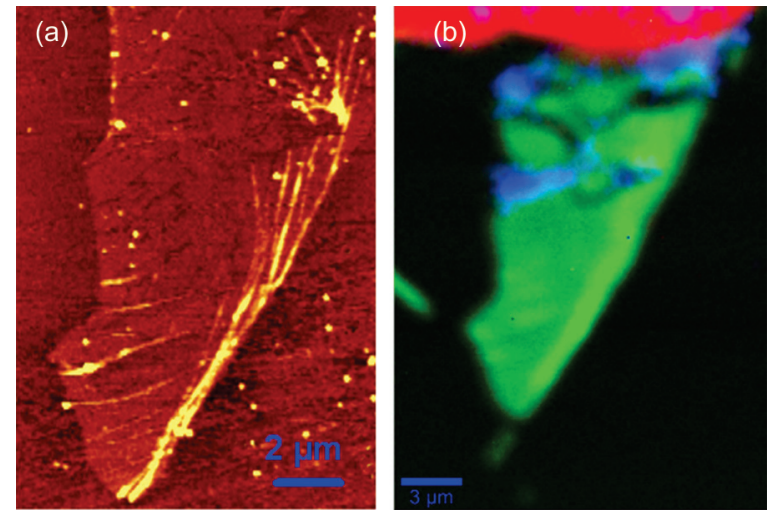

FIG. 9. (Color online) (a) AFM picture of graphene on $\mathrm{CaF}_{2}$; (b) Raman map of the flake: the green area corresponds to a defect-free graphene, while the blue area corresponds to defected graphene. The red area is thick graphite. 

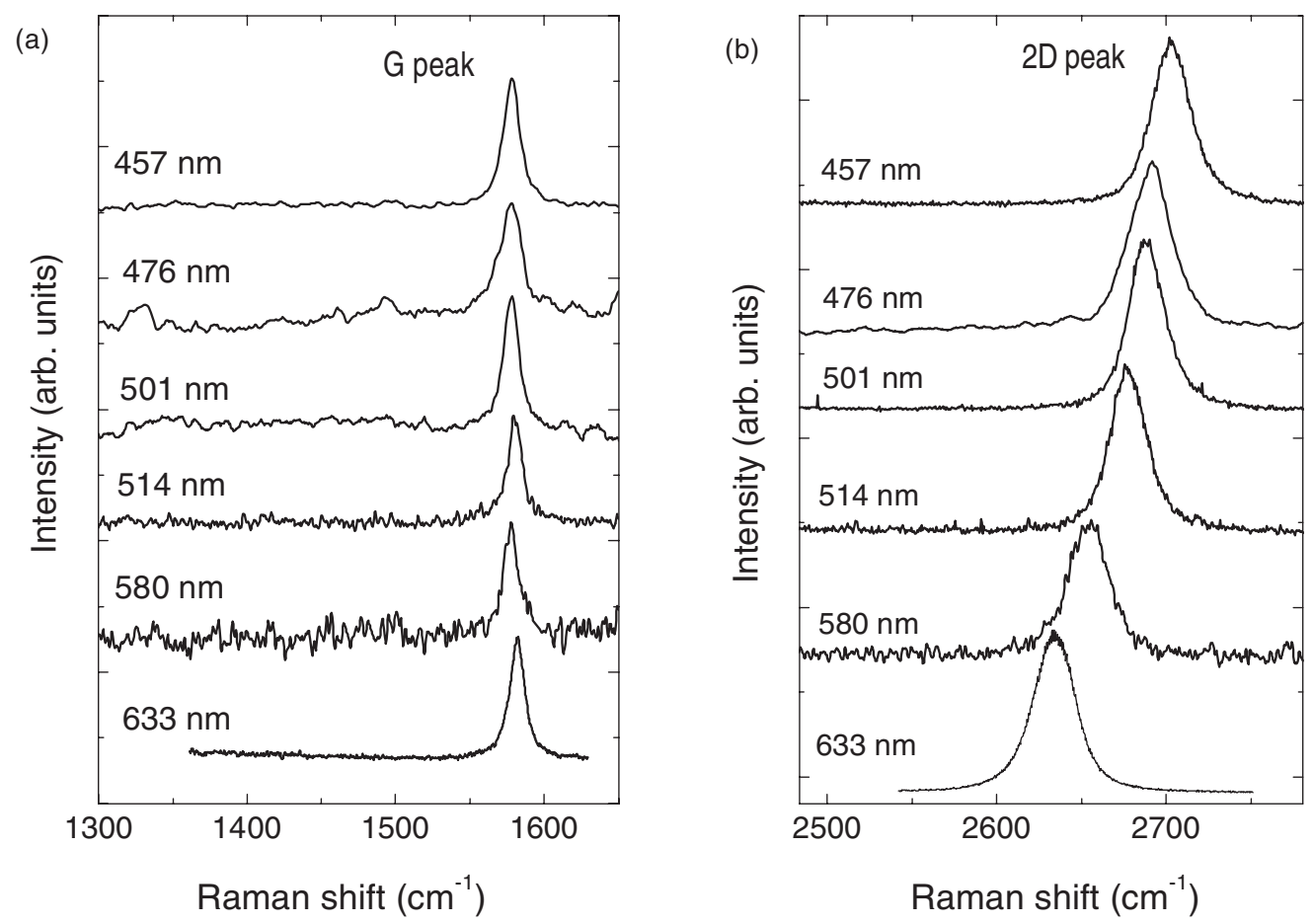

FIG. 10. (a) First and (b) second order Raman spectra of graphene on $\mathrm{CaF}_{2}$, measured at different excitation wavelengths.

The different energy dependence between the $G$ and $2 D$ peaks is a fingerprint of their different activation mechanisms: the $2 D$ peak involves resonant contributions, in agreement with recent measurements on intercalated graphite, ${ }^{86}$ while the $G$ peak cannot be described by a single-resonance model, in agreement with recent results. ${ }^{77,78}$ Note that the exact form of a resonance is determined by the phase and functional dependence of the matrix elements and the frequencies of the resonances. ${ }^{60}$

By using the data in Fig. 11, we can now calculate the Raman scattering efficiency of the $G$ and $2 D$ peaks for SLG, by using Eq. (12). At $2.41 \mathrm{eV} A(G)_{\mathrm{SLG}} /$ $A\left(\mathrm{CaF}_{2}\right) \sim 0.4$ and $A(2 D)_{\mathrm{SLG}} / A\left(\mathrm{CaF}_{2}\right) \sim 3.2$, so from

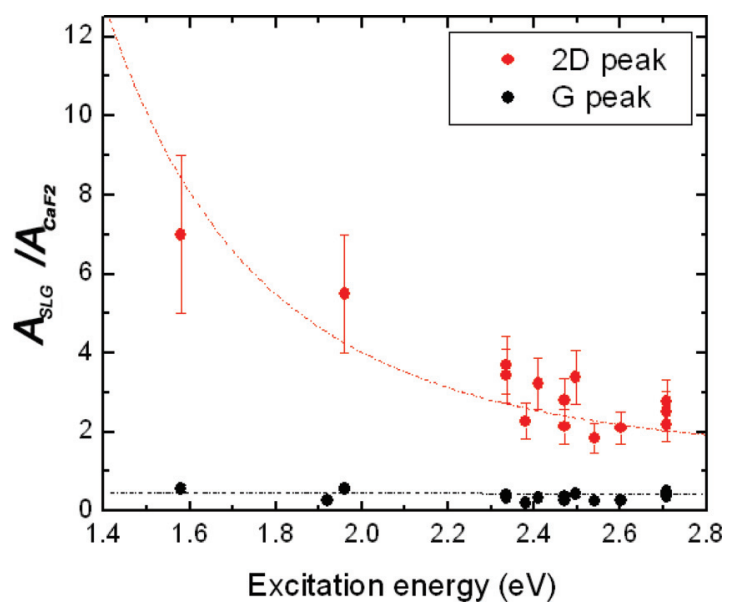

FIG. 11. (Color online) Areas of $G$ and $2 D$ peaks of graphene on $\mathrm{CaF}_{2}$ measured at different excitation energies and normalized to the $\mathrm{CaF}_{2}$ Raman peak area.
Eq. (12) we get $d S / d \Omega(G)_{\mathrm{SLG}} \sim 200 \times 10^{-5} \mathrm{~m}^{-1} \mathrm{Sr}^{-1}$ and $d S / d \Omega(G)(2 D)_{\mathrm{SLG}} \sim 1580 \times 10^{-5} \mathrm{~m}^{-1} \mathrm{Sr}^{-1}$.

We then consider SCG. Figure 12 plots $A(G)_{\mathrm{SCG}} / A\left(\mathrm{CaF}_{2}\right)$ and $A(2 D)_{\mathrm{SCG}} / A\left(\mathrm{CaF}_{2}\right)$. The energy dependence of the Raman peaks areas of SLG and SCG is the same, i.e., the $G$ peak Raman tensor is constant in the visible range, in contrast to the $2 D$ Raman tensor, in agreement with previous measurements on disordered graphite. ${ }^{52}$ For SCG we get $A(G)_{\mathrm{SCG}} / A\left(\mathrm{CaF}_{2}\right) \sim 4 \pm 1.2$ at $2.41 \mathrm{eV}$, so $d S / d \Omega(G)_{\mathrm{SCG}} \sim$ $100 \times 10^{-5} \mathrm{~m}^{-1} \mathrm{Sr}^{-1}$. This value is smaller than that reported in Ref. 51. However, taking into account that Ref. 51 measured HOPG and no spectrometer sensitivity correction was mentioned, we think that this difference is reasonable.

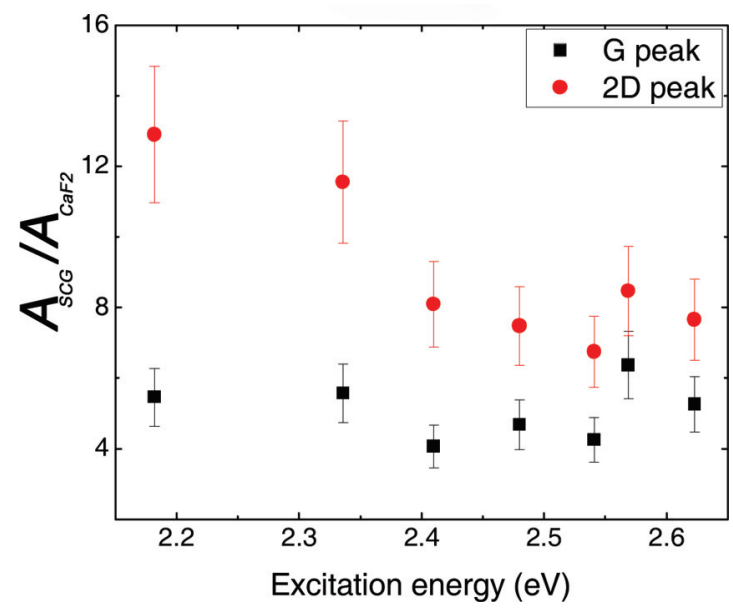

FIG. 12. (Color online) Areas of $G$ and $2 D$ peaks of graphite measured at different excitation energies and normalized to the $\mathrm{CaF}_{2}$ Raman peak area. 
TABLE I. Raman scattering efficiency of the $G$ peak of graphene and graphite, measured at $2.41 \mathrm{eV}$, compared to the Raman cross section measured for other materials. The units of $S$ are $10^{-5} \mathrm{~m}^{-1} \mathrm{Sr}^{-1}$. Note that $d S / d \Omega$ depends on the scattering geometry and temperature.

\begin{tabular}{|c|c|c|c|}
\hline & Graphene & Graphite & $\mathrm{CaF}_{2}$ \\
\hline$n$ & 2.69 & 2.69 & 1.437 \\
\hline$L(\mathrm{~nm})$ & 0.33 & 15 & 1000 \\
\hline$d S / d \Omega(G)$ & $\sim 200$ & $\sim 100$ & \\
\hline$d S / d \Omega(G)^{51}$ & & $300-400$ & \\
\hline$d S / d \Omega(G)^{50}$ & & 800 & \\
\hline$d S / d \Omega(\text { diamond) })^{71} 1332 \mathrm{~cm}^{-1}$ & & $6-5$ & \\
\hline$d S / d \Omega(\text { silicon })^{51} 521 \mathrm{~cm}^{-1}$ & & $500-700$ & \\
\hline$d S / d \Omega(\mathrm{c}-\mathrm{BN})^{71} 1332 \mathrm{~cm}^{-1}$ & & $0.1 \pm 0.2$ & \\
\hline$d S / d \Omega(\mathrm{h}-\mathrm{BN})^{71} 1332 \mathrm{~cm}^{-1}$ & & $20 \pm 10$ & \\
\hline$d S / d \Omega(\mathrm{GaAs})^{65} 267 \mathrm{~cm}^{-1}$ & & 950 & \\
\hline$d S / d \Omega(\mathrm{KI})$ second order ${ }^{93}$ & & 0.21 & \\
\hline$d S / d \Omega(\mathrm{KBr})$ second order ${ }^{93}$ & & 0.088 & \\
\hline$d S / d \Omega\left(\mathrm{SrTiO}_{3}\right)$ second order ${ }^{93}$ & & 172 & \\
\hline
\end{tabular}

In any case, the order of magnitude is the same. Table I compares $d S / d \Omega$ of the Raman peaks of graphene with the values reported for graphite and other materials.

Note the polarization dependence of the $G$ and $2 D$ peaks. The $G$ peak corresponds to a phonon with $E_{2 g}$ symmetry. Then, from group theory, we expect its intensity not to change with polarization. ${ }^{104}$ In contrast, the $2 D$ peak, being an overtone, always contains an $A_{1}$ symmetry. Then, from group theory we expect $A(2 D)$ to strongly change with polarization, i.e.,

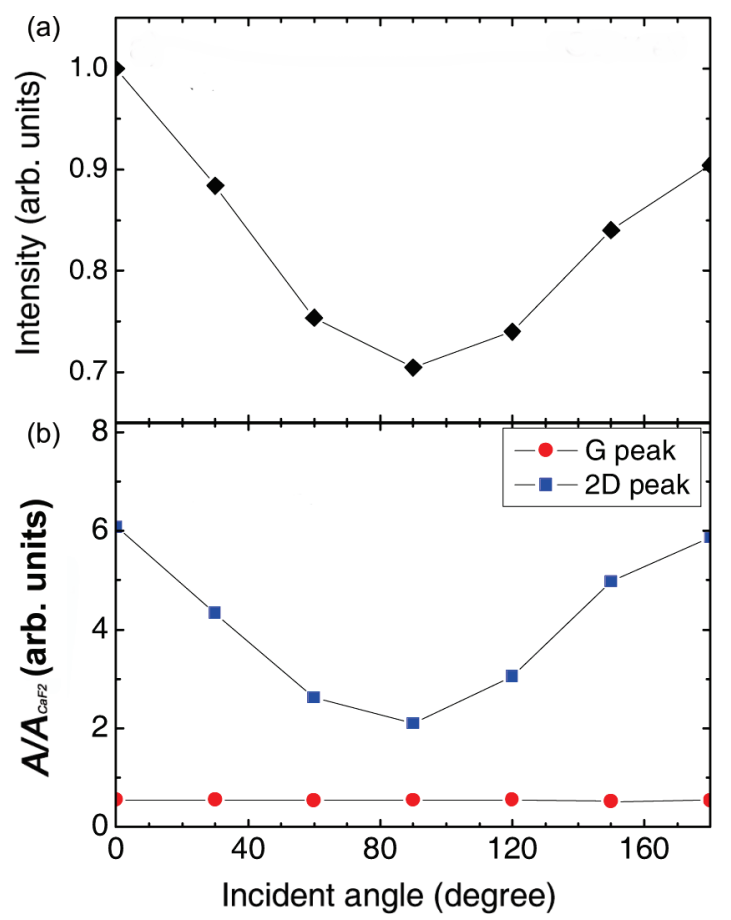

FIG. 13. (Color online) (a) Sensitivity of our Dilor spectrometer on the polarization angle; (b) dependence of $G$ and $2 D$ peak areas on polarization, after correction for the spectrometer sensitivity.

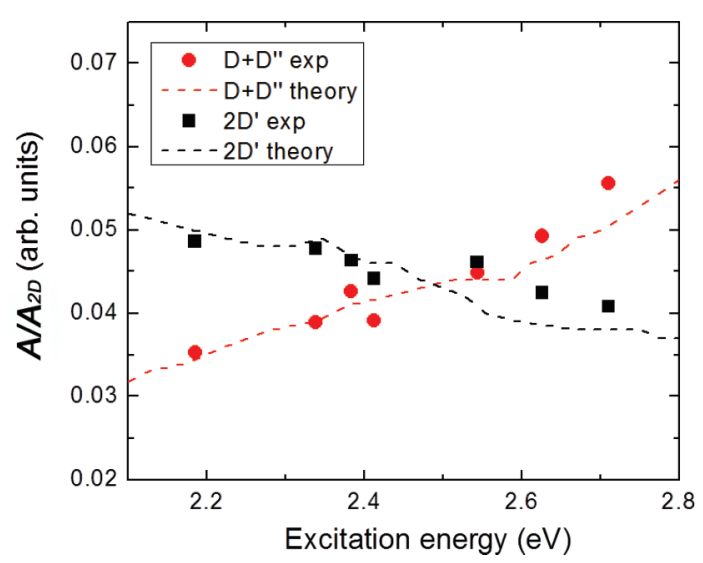

FIG. 14. (Color online) Comparison of the $D+D^{\prime \prime}$ and $2 D^{\prime}$ peak areas to the area of the $2 D$ peak. Theoretical data from Ref. 47 .

the maximum area should be obtained in the configuration which measures the diagonal components of the Raman tensor. ${ }^{79}$ We rotated $\mathrm{CaF}_{2}$, SLG, and SCG, but we did not observe any change in $A(G)$ and $A(2 D)$. This shows that the Raman peaks areas do not depend on the angle between the incident polarization and the crystal lattice, as discussed in Ref. 104: $A(2 D)$ depends only on the relative angle between incident and scattered polarization, no matter the incident polarization angle. We then used a Fresnel rhomb in order to rotate the polarization of the incident light. Now, we have to take into account that the whole spectrometer (grating and beam splitter) is sensitive to the polarization direction. Figure 13(a) shows the dependence of the spectrometer on the polarization, obtained by measuring the $\mathrm{CaF}_{2}$ intensity as a function of the incident polarization. Figure 13(b) plots $A(G)$ and $A(2 D)$ as a function of the polarization angle, after correction for the spectrometer sensitivity. This shows that the beam splitter acts as an analyzer. This explains why, even after correcting $A(G)$ and $A(2 D)$ for the polarization dependence, $A(2 D)$ changes with polarization. Indeed, in order to avoid effects arising from the spectrometer sensitivity on polarization it is necessary to insert a half- $\lambda$ plate before beam splitter and detector, as done in Ref. 104. Figure 13 shows that under our configuration, i.e., without introducing any external polarizer/analyzer, our spectrometer measures the maximum $A(2 D)$.

It is also interesting to compare the relative intensity of the two-phonon lines, such as $2 D, 2 D^{\prime}$, and $D+D^{\prime \prime}$, measured for SLG on $\mathrm{CaF}_{2}$ as a function of excitation energy. The $D+D^{\prime \prime}$ peak lies at $\sim 2450 \mathrm{~cm}^{-1}$, and it is a combination between $D$ and the $D^{\prime \prime}$ peak at $\sim 1100 \mathrm{~cm}^{-1} .^{42,105-109}$ The relative areas of these peaks shows a peculiar dependence on excitation energy: $A\left(2 D^{\prime}\right) / A(2 D)$ decreases for increasing excitation energy, while $A\left(D+D^{\prime \prime}\right) / A(2 D)$ increases, see Fig. 14. This further confirms the importance of resonant processes on the two-phonon lines area.

\section{CONCLUSIONS}

We presented a detailed analysis of the Raman intensities of graphene. We measured the absolute Raman efficiency 
of graphene and graphite in the visible range by sample substitution. We showed how the Raman scattering efficiency of graphene changes with the number of layers, the type of substrate, and the incident laser frequency. In particular, we showed that the Raman intensity of graphene is strongly modulated by the $\mathrm{Si} / \mathrm{SiO}_{x}$ substrate, as confirmed by depositing graphene on a different substrate $\left(\mathrm{CaF}_{2}\right)$.

By using the sample-substitution method, we measured the absolute Raman efficiency of graphene. The $G$ peak Raman scattering efficiency is $\sim 200 \times 10^{-5} \mathrm{~m}^{-1} \mathrm{~S}^{-1}$. For the $2 D$ peak the Raman scattering efficiency is one order of magnitude bigger than that of the $G$ peak, at $2.41 \mathrm{eV}$. Furthermore, its absolute value strongly depends on light polarization. The high Raman scattering efficiency of the $2 D$ peak and its dependence on the excitation energy further confirm the resonant nature of the Raman process giving rise to this peak.

\section{ACKNOWLEDGMENTS}

The authors acknowledge useful discussions with D. M. Basko, L. G. Cancado, M. Kalbac, and S. Reich. P.K., C.C., and I.A.V. acknowledge funding by the Alexander von Humboldt Foundation in the framework of the Sofja Kovalevskaja Award, endowed by the Federal Ministry of Education and Research of Germany. A.C.F. acknowledges funding from the Royal Society, the ERC grant NANOPOTS, EPSRC grants EP/G042357/1, EP/K01711X/1, EP/K017144/1, EU grants RODIN, GENIUS, MEM4WIN and CareRAMM.
${ }^{1}$ K. S. Novoselov, A. K. Geim, S. V. Morozov, D. Jiang, Y. Zhang, S. V. Dubonos, I. V. Grigorieva, and A. A. Firsov, Science 306, 666 (2004).

${ }^{2}$ A. K. Geim and K. S. Novoselov, Nat. Mater. 6, 183 (2007).

${ }^{3}$ A. H. Castro Neto, F. Guinea, N. M. R. Peres, K. S. Novoselov, and A. K. Geim, Rev. Mod. Phys. 81, 109 (2009).

${ }^{4}$ J. C. Charlier, P. C. Eklund, J. Zhu, and A. C. Ferrari, Topics Appl. Phys. 111, 673 (2008).

${ }^{5}$ K. S. Novoselov, A. K. Geim, S. V. Morozov, D. Jiang, M. I. Katsnelson, I. V. Grigorieva, S. V. Dubonos, and A. A. Firsov, Nature (London) 438, 197 (2005).

${ }^{6}$ Y. Zhang, Y. W. Tan, H. L. Stormer, and P. Kim, Nature (London) 438, 201 (2005).

${ }^{7}$ F. Bonaccorso, A. Lombardo, T. Hasan, Z. Sun, L. Colombo, and A. C. Ferrari, Mater. Today 15, 564 (2012).

${ }^{8}$ K. S. Novoselov, Z. Jiang, Y. Zhang, S. V. Morozov, H. L. Stormer, U. Zeitler, J. C. Maan, G. S. Boebinger, P. Kim, and A. K. Geim, Science 315, 1379 (2007).

${ }^{9}$ S. V. Morozov, K. S. Novoselov, M. I. Katsnelson, F. Schedin, D. C. Elias, J. A. Jaszczak, and A. K. Geim, Phys. Rev. Lett. 100, 016602 (2008).

${ }^{10}$ X. Du, I. Skachko, A. Barker, and E. Y. Andrei, Nature Nano. 3, 491 (2008).

${ }^{11}$ K. I. Bolotin, K. J. Sikes, J. Hone, H. L. Stormer, and P. Kim, Phys. Rev. Lett. 101, 096802 (2008).

${ }^{12}$ M. Y. Han, B. Özyilmaz, Y. Zhang, and P. Kim, Phys. Rev. Lett. 98, 206805 (2007).

${ }^{13}$ Z. Chen, Y. M. Lin, M. Rooks, and P. Avouris, Physica E 40, 228 (2007).

${ }^{14}$ Y. Zhang, J. P. Small, W. V. Pontius, and P. Kim, Appl. Phys. Lett. 86, 073104 (2005)

${ }^{15}$ M. C. Lemme, T. J. Echtermeyer, M. Baus, and H. Kurz, IEEE Electron Device Lett. 28, 282 (2007).

${ }^{16}$ Y. M. Lin, K. A. Jenkins, A. Valdes-Garcia, J. P. Small, D. B. Farmer, and P. Avouris, Nano Lett. 9, 422 (2009).

${ }^{17}$ J. S. Bunch, A. M. van der Zande, S. S. Verbridge, I. W. Frank, D. M. Tanenbaum, J. M. Parpia, H. G. Craighead, and P. L. McEuen, Science 315, 490 (2007).

${ }^{18}$ P. Blake, P. D. Brimicombe, R. R. Nair, T. J. Booth, D. Jiang, F. Schedin, L. A. Ponomarenko, S. V. Morozov, H. F. Gleeson, E. W. Hill, A. K. Geim, and K. S. Novoselov, Nano Lett. 8, 1704 (2008).
${ }^{19}$ Y. Hernandez, V. Nicolosi, M. Lotya, F. Blighe, Z. Sun, S. De, I. T. McGovern, B. Holland, M. Byrne, Y. Gunko, J. Boland, P. Niraj, G. Duesberg, S. Krishnamurti, R. Goodhue, J. Hutchison, V. Scardaci, A. C. Ferrari, and J. N. Coleman, Nature Nano. 3, 563 (2008).

${ }^{20}$ G. Eda, G. Fanchini, and M. Chhowalla, Nature Nano. 3, 270 (2008).

${ }^{21}$ Z. Sun, T. Hasan, F. Torrisi, D. Popa, G. Privitera, F. Wang, F. Bonaccorso, D. M. Basko, and A. C. Ferrari, ACS Nano 4, 803 (2010).

${ }^{22}$ F. Bonaccorso, Z. Sun, T. Hasan, and A. C. Ferrari, Nat. Photon. 4, 611 (2010).

${ }^{23}$ T. Gokus, R. R. Nair, A. Bonetti, M. Bohmler, A. Lombardo, K. S. Novoselov, A. K. Geim, A. C. Ferrari, and A. Hartschuh, ACS Nano 3, 3963 (2009).

${ }^{24}$ T. Mueller, F. Xia, and P. Avouris, Nat. Photon. 4, 297 (2010).

${ }^{25}$ A. C. Ferrari, J. C. Meyer, V. Scardaci, C. Casiraghi, M. Lazzeri, F. Mauri, S. Piscanec, D. Jiang, K. S. Novoselov, S. Roth, and A. K. Geim, Phys. Rev. Lett. 97, 187401 (2006).

${ }^{26}$ C. Casiraghi, A. Hartschuh, E. Lidorikis, H. Qian, H. Harutyunyan, T. Gokus, K. S. Novoselov, and A. C. Ferrari, Nano Lett. 7, 2711 (2007).

${ }^{27}$ P. Blake, E. W. Hill, A. H. Castro Neto, K. S. Novoselov, D. Jiang, R. Yang, T. J. Booth, and A. K. Geim, Appl. Phys. Lett. 91, 063124 (2007).

${ }^{28}$ A. C. Ferrari, Solid State Commun. 143, 47 (2007).

${ }^{29}$ C. Casiraghi, in Raman Spectroscopy of Graphene in Spectroscopic Properties of Inorganic and Organometallic Compounds. Techniques, Materials and Applications, edited by J. Yarwood, R. Douthwaite, and S. Duckett (RCS Publishing, Cambridge, 2012), Vol. 43, p. 29.

${ }^{30}$ S. Pisana, M. Lazzeri, C. Casiraghi, K. S. Novoselov, A. K. Geim, A. C. Ferrari, and F. Mauri, Nat. Mater. 6, 198 (2007).

${ }^{31}$ A. Das, S. Pisana, S. Piscanec, B. Chakraborty, S. K. Saha, U. V. Waghmare, R. Yang, H. R. Krishnamurhthy, A. K. Geim, A. C. Ferrari, and A. K. Sood, Nature Nano. 3, 210 (2008).

${ }^{32}$ C. Casiraghi, S. Pisana, K. S. Novoselov, A. K. Geim, and A. C. Ferrari, Appl. Phys. Lett. 91, 233108 (2007).

${ }^{33}$ C. Casiraghi, A. Hartschuh, H. Qian, S. Piscanec, C. Georgi, A. Fasoli, K. S. Novoselov, D. M. Basko, and A. C. Ferrari, Nano Lett. 9, 1433 (2009). 
${ }^{34}$ C. Casiraghi, Phys. Status Solidi RRL 3, 175 (2009).

${ }^{35}$ D. C. Elias, R. R. Nair, T. M. G. Mohiuddin, S. V. Morozov, P. Blake, M. P. Halsall, A. C. Ferrari, D. W. Boukhvalov, M. I. Katsnelson, A. K. Geim, and K. S. Novoselov, Science 323, 610 (2009).

${ }^{36}$ T. M. G. Mohiuddin, A. Lombardo, R. R. Nair, A. Bonetti, G. Savini, R. Jalil, N. Bonini, D. M. Basko, C. Galiotis, N. Marzari, K. S. Novoselov, A. K. Geim, and A. C. Ferrari, Phys. Rev. B 79, 205433 (2009).

${ }^{37}$ J. Zabel, R. R. Nair, A. Ott, T. Georgiou, A. K. Geim, K. S. Novoselov, and C. Casiraghi, Nano Lett. 12, 617 (2012).

${ }^{38}$ E. H. Martins Ferreira, M. V. O. Moutinho, F. Stavale, M. M. Lucchese, R. B. Capaz, C. A. Achete, and A. Jorio, Phys. Rev. B 82, 125429 (2010)

${ }^{39}$ L. G. Cancado, A. Jorio, E. H. Martins Ferreira, F. Stavale, C. A. Achete, R. B. Capaz, M. V. O. Moutinho, A. Lombardo, T. S. Kulmala, and A. C. Ferrari, Nano Lett. 11, 3190 (2011).

${ }^{40}$ A. Eckmann, A. Felten, A. Mishchenko, L. Britnell, R. Krupke, K. S. Novoselov, and C. Casiraghi, Nano Lett. 12, 3925 (2012).

${ }^{41}$ P. H. Tan, W. P. Han, W. J. Zhao, Z. H. Wu, K. Chang, H. Wang, Y. F. Wang, N. Bonini, N. Marzari, N. Pugno, G. Savini, A. Lombardo, and A. C. Ferrari, Nat. Mater. 11, 294 (2012).

${ }^{42}$ A. C. Ferrari and D. M. Basko, Nat. Nanotechnol. 8, 235 (2013).

${ }^{43}$ S. Piscanec, M. Lazzeri, F. Mauri, A. C. Ferrari, and J. Robertson, Phys. Rev. Lett. 93, 185503 (2004).

${ }^{44}$ D. M. Basko, S. Piscanec, and A. C. Ferrari, Phys. Rev. B 80, 165413 (2009).

${ }^{45}$ C. Thomsen and S. Reich, Phys. Rev. Lett. 85, 5214 (2000).

${ }^{46}$ D. M. Basko, Phys. Rev. B 78, 125418 (2008).

${ }^{47}$ P. Venezuela, M. Lazzeri, and F. Mauri, Phys. Rev. B 84, 035433 (2011).

${ }^{48}$ J. M. Calleja, J. Kuhl, and M. Cardona, Phys. Rev. B 17, 876 (1978).

${ }^{49}$ A. K. McQuillan, W. R. L. Clemens, and B. P. Stoicheff, Phys. Rev. A 1, 628 (1970).

${ }^{50}$ K. Sinha and J. Menendez, Phys. Rev. B 41, 10845 (1990).

${ }^{51}$ N. Wada and S. A. Solin, Physica B 105, 353 (1981).

${ }^{52}$ L. G. Cancado, A. Jorio, and M. A. Pimenta, Phys. Rev. B 76, 064304 (2007).

${ }^{53}$ J. D. Lorentzen, S. Guha, J. Menendez, P. Giannozzi, and S. Baroni, Chem. Phys. Lett. 270, 129 (1997).

${ }^{54}$ J. Wagner, M. Ramsteiner, Ch. Wild, and P. Koidl, Phys. Rev. B 40, 1817 (1989).

${ }^{55}$ M. Ramsteiner and J. Wagner, Appl. Phys. Lett. 51, 1355 (1987).

${ }^{56}$ C. Casiraghi, Diamond Relat. Mater. 20, 120 (2011).

${ }^{57}$ S. R. Sails, D. J. Gardiner, M. Bowden, J. Savage, and D. Rodway, Diamond Relat. Mater. 5, 589 (1996).

${ }^{58}$ H. Telg, J. Maultzsch, S. Reich, and C. Thomsen, Phys. Rev. B 74, 115415 (2006)

${ }^{59}$ J. E. Bohn, P. G. Etchegoin, E. C. Le Ru, R. Xiang, S. Chiashi, and S. Maruyama, ACS Nano 4, 3466 (2010).

${ }^{60}$ M. Cardona, in Light Scattering in Solids II, edited by M. Cardona and G. Güntherodts, Topics in Applied Physics Vol. 50 (Springer, Berlin, 1982), p. 19.

${ }^{61}$ Y. Prior and H. Vogt, Phys. Rev. B 19, 5388 (1979).

${ }^{62}$ C. A. Arguello, D. L. Rosseau, and S. P. S. Porto, Phys. Rev. 181, 1351 (1969).

${ }^{63}$ C. Trallero-Giner, K. Kunc, and K. Syassen, Phys. Rev. B 73, 205202 (2006)

${ }^{64}$ K. Aoki and M. Cardona, Phys. Status Solidi B 122, 441 (1984).
${ }^{65}$ M. H. Grimsditch, D. Olego, and M. Cardona, Phys. Rev. B 20, 1758 (1979)

${ }^{66}$ F. Cerdeira, W. Dreybrodt, and M. Cardona, Solid State Commun. 10, 591 (1972).

${ }^{67}$ R. H. Callender, S. S. Sussman, M. Selders, and R. K. Chang, Phys. Rev. B 7, 3788 (1973).

${ }^{68}$ W. Limmer, H. Leiderer, K. Jakob, W. Gebhardt, W. Kauschke, A. Cantarero, and C. Trallero-Giner, Phys. Rev. B 42, 11325 (1990).

${ }^{69}$ H. Leiderer, M. Silberbauer, S. Bauer, W. Limmer, and G. Gebhardt, J. Phys.: Condens. Matter 6, 6057 (1994).

${ }^{70}$ J. B. Renucci, R. N. Tyte, and M. Cardona, Phys. Rev. B 11, 3885 (1975).

${ }^{71}$ S. Reich, A. C. Ferrari, R. Arenal, A. Loiseau, I. Bello, and J. Robertson, Phys. Rev. B 71, 205201 (2005).

${ }^{72}$ R. E. Shroder, R. J. Nemanich, and J. T. Glass, Phys. Rev. B 41, 3738 (1990).

${ }^{73}$ A. C. Ferrari and J. Robertson, eds., Phil. Trans. R. Soc. A 362, 2267 (2004)

${ }^{74}$ F. Tuinstra and J. L. Koenig, J. Chem. Phys. 53, 1126 (1970).

${ }^{75}$ A. C. Ferrari and J. Robertson, Phys. Rev. B 61, 14095 (2000); 64, 075414 (2001).

${ }^{76}$ D. M. Basko, New J. Phys. 11, 095011 (2009).

${ }^{77}$ M. Kalbac, A. Reina-Cecco, H. Farhat, J. Kong, L. Kavan, and M. S. Dresselhaus, ACS Nano 10, 6055 (2010).

${ }^{78}$ C. Chen, C. Park, B. W. Boudouris, J. Horng, B. Geng, C. Girit, A. Zettl, M. F. Crommie, R. A. Segalman, S. G. Louie, and F. Wang, Nature (London) 471, 617 (2011).

${ }^{79}$ R. J. Nemanich and S. A. Solin, Phys. Rev. B 20, 392 (1979).

${ }^{80}$ R. Vidano and D. B. Fishbach, J. Am. Ceram. Soc. 61, 13 (1978).

${ }^{81}$ P. Lespade, A. Marchand, M. Couzi, and F. Cruege, Carbon 4-5, 375 (1984).

${ }^{82}$ Y. Wang, D. C. Alsmeyer, and R. L. McCreery, Chem. Mater. 2, 557 (1990).

${ }^{83}$ I. Póksik, M. Hundhausen, M. Koós, and L. Ley, J. Non-Cryst. Solids 227-230, 1083 (1998).

${ }^{84}$ K. Sato, R. Saito, Y. Oyama, J. Jiang, L. G. Cancado, M. A. Pimenta, A. Jorio, Ge. G. Samsonidze, G. Dresselhaus, and M. S. Dresselhaus, Chem. Phys. Lett. 427, 117 (2006).

${ }^{85}$ J. Maultzsch, H. Telg, S. Reich, and C. Thomsen, Phys. Rev. B 72, 205438 (2005).

${ }^{86}$ W. J. Zhao, P. H. Tan, J. Liu, and A. C. Ferrari, J. Am. Chem. Soc. 133, 5941 (2011).

${ }^{87}$ K. S. Novoselov, D. Jiang, F. Schedin, T. J. Booth, V. V. Khotkevich, S. V. Morozov, and A. K. Geim, Proc. Natl. Acad. Sci. USA 102, 10451 (2005).

${ }^{88}$ S. Akcoltekin, M. El Kharrazi, B. Kohler, A. Lorke, and M. Schleberger, Nanotechnol. 20, 155601 (2009).

${ }^{89}$ H. G. Poole, J. Chem. Soc. 245 (1946).

${ }^{90}$ M. H. Grimsditch and A. K. Ramdas, Phys. Rev. B 11, 3139 (1975).

${ }^{91}$ M. Grimsditch and M. Cardona, Phys. Status Solidi B 102, 155 (1980).

${ }^{92}$ P. Gallo, V. Mazzucurati, G. Ruocco, and G. Signorelli, Phys. Rev. B 43, 14268 (1991).

${ }^{93}$ M. H. Grimsditch, Solid State Commun. 25, 389 (1978).

${ }^{94}$ M. V. Klein, in Light Scattering in Solids I, 2nd ed., edited by M. Cardona, Topics in Applied Physics Vol. 8 (Springer, Berlin, 1983), p. 147. 
${ }^{95}$ C. Trallero-Giner, A. Cantarero, M. Cardona, and M. Mora, Phys. Rev. B 45, 6601 (1992).

${ }^{96}$ I. Loa, S. Gronemeyer, C. Thomsen, O. Ambacher, D. Schikora, and D. J. As, J. Raman Spectrosc. 29, 291 (1998).

${ }^{97}$ E. Hecht, Optics (Addison-Wesley, Redwood City, CA, 1998).

${ }^{98}$ M. Grimsditch, M. Cardona, J. M. Calleja, and F. Meseguer, J. Raman Spectrosc. 10, 77 (1981).

${ }^{99}$ R. R. Nair, P. Blake, A. N. Grigorenko, K. S. Novoselov, T. J. Booth, T. Stauber, N. M. R. Peres, and A. K. Geim, Science 320, 1308 (2008).

${ }^{100}$ V. G. Kravets, A. N. Grigorenko, R. R. Nair, P. Blake, S. Anissimova, K. S. Novoselov, and A. K. Geim, Phys. Rev. B 81, 155413 (2010).

${ }^{101}$ A. B. Djurisic and E. Herbert Li, J. Appl. Phys. 85, 7404 (1999).
${ }^{102}$ C. Casiraghi, Phys. Rev. B 80, 233407 (2009).

${ }^{103}$ S. Berciaud, S. Ryu, L. E. Brus, and T. F. Heinz, Nano Lett. 9, 346 (2009).

${ }^{104}$ D. Yoon, H. Moon, Y.-W. Son, G. Samsonidze, B. H. Park, J. B. Kim, Y.-P. Lee, and H. Cheong, Nano Lett. 8, 4270 (2008).

${ }^{105}$ P. H. Tan, Y. M. Deng, and Q. Zhao, Phys. Rev. B 58, 5435 (1998).

${ }^{106}$ P. H. Tan, L. An, L. Q. Liu, Z. X. Guo, R. Czerw, D. L. Carroll, P. M. Ajayan, N. Zhang, and H. L. Guo, Phys. Rev. B 66, 245410 (2002).

${ }^{107}$ P. H. Tan, C. Y. Hu, J. Dong, W. C. Shen, and B. F. Zhang, Phys. Rev. B 64, 214301 (2001).

${ }^{108}$ Y. Kawashima and G. Katagiri, Phys. Rev. B 52, 10053 (1995).

${ }^{109}$ P. May, M. Lazzeri, P. Venezuela, F. Herziger, G. Callsen, J. S. Reparaz, A. Hoffmann, F. Mauri, and J. Maultzsch, Phys. Rev. B 87, 075402 (2013). 